\title{
Novel missense mutation in the caveolin-3 gene in a Belgian family with rippling muscle disease
}

\author{
P Y K Van den Bergh, J M Gérard, J A Elosegi, M U Manto, C Kubisch, B G H Schoser
}

J Neurol Neurosurg Psychiatry 2004;75:1349-1351. doi: 10.1136/jnnp.2003.028217

Rippling muscle disease (RMD) is a rare muscle disorder characterised by muscle stiffness, exercise induced myalgia, and cramp-like sensations. It is genetically heterogeneous and can be acquired, but most cases show autosomal dominant inheritance due to mutations in the caveolin-3 (CAV3) gene. We report a novel heterozygous missense mutation in CAV3 in a Belgian family with autosomal dominant RMD.

A 40 year old woman complained of fatigue, exercise induced muscle pain, and muscle cramps since the age of 35 . Neurological examination revealed percussion induced rapid muscle contractions (PIRCs) and localised muscle mounding on percussion; muscle rippling was not observed. Creatine kinase (CK) was elevated but electromyography and nerve conduction studies were normal. Fluorescence immunohistochemistry revealed reduced caveolin-3 and dysferlin staining in a quadriceps muscle biopsy. Western blot analysis confirmed severely reduced caveolin-3 levels, whereas dysferlin was normal. Sequence analysis of the two coding exons of CAV3 revealed a hitherto unreported heterozygous C82A transversion in the first exon, predicting a Pro28Thr amino acid exchange. Thr patient's first degree relatives did not present with neuromuscular complaints, but PIRCs, muscle mounding, and muscle rippling were found in the mother, who also carried the CAV3 mutation.

$\mathrm{R}$ ippling muscle disease (RMD) is a rare muscle disorder, characterised by muscle stiffness, exercise induced myalgia, and cramp-like sensations. ${ }^{1-3}$ Because of mechanical hyperirritability, tapping on muscle or stretching it leads to electrically silent muscle contractions, clinically manifested by percussion induced rapid muscle contractions (PIRCs), localised, often painful, muscle mounding, and rolling movements across muscle groups ("rippling"). Muscle hypertrophy, especially of the calves, and raised levels of serum creatine kinase (CK) are frequently found. RMD is genetically heterogeneous and can be acquired, but most cases show autosomal dominant inheritance and are associated with mutations in the caveolin-3 (CAV3) gene. ${ }^{4}{ }^{5} \mathrm{We}$ report the first Belgian family with RMD, in whom we discovered a novel mutation in CAV3.

\section{CASE REPORT}

\section{Propositus}

A 40 year old white cleaning lady of Belgian-Italian descent complained of fatigue, pain in the neck and shoulders, and muscle stiffness since the age of 35 . Exercise induced muscle pain and cramps in all four limbs interfered with running, walking, lifting buckets, and wringing cloths. The myalgia was described as a tearing-like sensation ("as if the skin were too small"). The patient was diagnosed as having fibromyalgia, which led to secondary depression and loss of her job. Muscle weakness and pigmenturia were absent. The medical history was remarkable for mild hypothyroidism, peptic oesophagitis, and tobacco related asthmatiform bronchitis with emphysema. At age 38, she had a neurological work-up. The cranial nerves, muscle bulk, muscle strength, and deep tendon reflexes were normal, and plantar responses were flexor. Tapping with the reflex hammer or with the finger on the muscles provoked an immediate, short lasting, forceful contraction and/or painful local mounding lasting for several seconds. PIRCs were most pronounced in the sternocleidomastoid, deltoid, biceps, brachioradialis, finger and wrist extensors, and anterior tibial muscles. Action or percussion myotonia was not observed.

Blood analysis showed raised serum CK ( 1184 IU/l, normal $<167$ IU/l) but was otherwise normal. An electrocardiogram (ECG) and heart ultrasound were normal and nerve conduction studies and electromyography (EMG) were unremarkable. There were no myotonic discharges. PIRCs were associated with bursts of normal motor unit action potentials. During muscle mounding after tapping, no electrical activity in the muscle was recorded. A computed tomography (CT) scan of muscle was normal. A quadriceps muscle biopsy showed increased variability in fibre size as well as mild atrophy and predominance of type $2 \mathrm{~B}$ fibres.

\section{Family}

The unrelated parents, both 61 years old, a 38 year old sister, and a 21 year old son had no complaints of neuromuscular symptoms. The Italian born father had had a stroke at 57 years from which he fully recovered. Neurological examination and serum CK were normal in all except the mother, in whom PIRCs and muscle mounding were as pronounced as in the propositus. Moreover, rolling movements ("rippling") across the quadriceps muscles were provoked by forceful tapping or stretching of the muscle. Serum CK was mildly elevated at $333 \mathrm{UI} / \mathrm{l}$ (normal <170 IU/1)

On the basis of these findings, the diagnosis of autosomal dominant RMD was proposed. The patient's symptoms were not progressive but they interfered significantly with most activities of daily living. Treatment with carbamazepine, diphantoin, topiramate, gabapentin, tricyclic antidepressants, venlafaxine, mexiletine, clonazepam, tramadol, dantrolene sodium, chlortalidone, and verapamil was unsuccessful.

\section{Investigations}

Muscle immunohistochemistry and Western blot analysis

Using standard procedures, immunofluorescence studies using Flousescein isothioayanate (FITC) labelled secondary

Abbreviations: CAV3, caveolin-3 gene; LGMDIC, limb girdle muscular dystrophy; nNOS, neuronal nitric acid synthase; PIRC, percussion induced rapid muscle contractions; RMD, rippling muscle disease 
antibodies were done on transverse, frozen muscle tissue sections with monoclonal antibodies against spectrin, dystrophin $1,2,3, \alpha-, \beta-, \gamma-$, and $\delta$-sarcoglycan, merosin $(80 \mathrm{kDa}$ and $300 \mathrm{kDa}$ ), dysferlin, and caveolin-3 (Novocastra, Newcastle-upon-Tyne, UK). Muscle proteins were separated by electrophoresis through a $7 \%$ (for dysferlin) or 12\% (for caveolin-3) polyacrylamide gel. Blots were incubated with dysferlin and caveolin-3 antibodies, followed by horseradish peroxidase labelled mouse anti-IgG antibodies, and developed with an electrochemiluminescent (ECL) detection system (Amersham Pharmacia Biotech, Uppsala, Sweden).

\section{Genetic analysis}

Genomic DNA was extracted from peripheral blood lymphocytes of the propositus, her parents, sister, and son. Polymerase chain reaction (PCR) products were subjected to direct sequencing of the two coding exons of CAV3 as previously described. ${ }^{4}$

\section{Results}

Caveolin-3 and dysferlin immunofluorescence was clearly reduced at the sarcolemma of all muscle fibres in the biopsy of the propositus as compared with the control (fig 1). Dystrophin, sarcoglycans, and merosin were normal. Western blot analysis showed almost complete absence ( $\sim 95 \%$ reduction) of caveolin-3, whereas dysferlin levels were normal (fig 1). Mutation analysis revealed a hitherto unreported heterozygous $\mathrm{C}>\mathrm{A}$ transversion at position 82 in the first exon of $C A V 3$, predicting a Pro28Thr amino acid exchange, in the propositus and her mother. This sequence variation was not found either in the other family members or in the 100 control chromosomes. Protein sequence alignment of all known caveolins from vertebrates shows that the proline residue at position 28 is perfectly conserved. ${ }^{6}$

\section{DISCUSSION}

We report the first Belgian family with autosomal dominant RMD due to a novel CAV3 mutation. The propositus presented

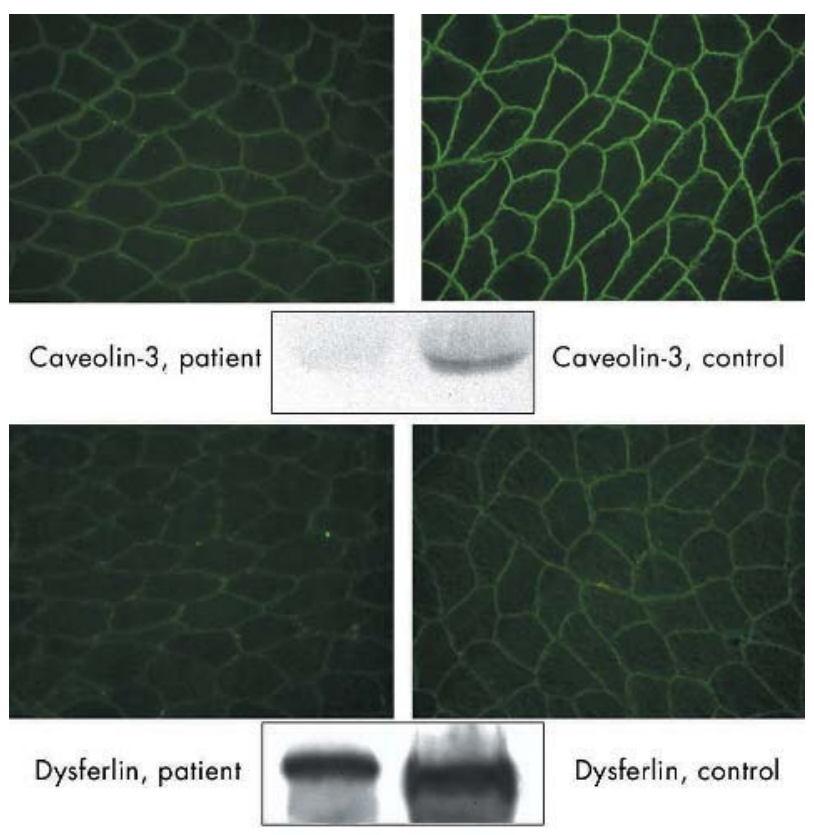

Figure 1 Results of the immunofluorescence tests and Western blotting analysis of the muscle biopsy of the patient compared with a normal control subject. Whereas in the patient, both caveolin-3 and dysferlin immunostaining were reduced, immunoblotting revealed that dysferlin levels were normal and that caveolin-3 was virtually absent. at age 40 with characteristic symptoms of muscle stiffness, exercise induced myalgia, and cramp-like sensations. Clinical signs consisted of PIRCs and percussion induced, electrically silent, painful muscle mounding. Muscle rippling was absent. According to Vorgerd et $a l,{ }^{3}$ late onset (after age 20) of the disorder occurs in $9 \%$ of cases and rippling, one of the triad of signs, is absent in approximately $40 \%$ of cases. Interestingly, the mother, who had no complaints, presented with the typical triad of PIRCs, mounding, and rippling. This illustrates that because $15 \%$ of RMD cases do not have neuromuscular complaints ${ }^{3}$ careful neurological examination of family members is of paramount importance in determining subclinical disease.

Nine missense mutations in CAV3 have been reported in RMD including the present report; seven of these are heterozygous ${ }^{578}$ and two are homozygous. ${ }^{5}$ In our family, the heterozygous C82A sequence variation in the first exon, predicting a Pro28Thr amino acid exchange, is most likely pathogenic because (i) it cosegregates with the disease in this family, (ii) it is associated with severely reduced caveolin-3 protein levels as suggested by the immunofluorescence studies and confirmed by Western blotting, (iii) it is not observed in control chromosomes, (iv) the exchanged amino acid is highly conserved in known vertebrate caveolins, and (v) a different mutation of the same amino acid (Pro28Leu) has been reported in a family with autosomal dominant hyperCKaemia. ${ }^{9}$

CAV3 mutations are not only associated with RMD-they were first reported in patients with autosomal dominant limb girdle muscular dystrophy (LGMDIC),,$^{10-14}$ and later in sporadic $^{15}$ and familial ${ }^{9}$ hyperCKaemia, and distal myopathy. ${ }^{16}{ }^{17}$ Genotype-phenotype correlations are highly speculative at present. A given mutation may lead to different phenotypes in different families and even within the same family. For instance, all caveolinopathy phenotypes have been reported for the Arg26Gln substitution. This particular caveolin-3 mutation was shown to be mostly retained at the level of the Golgi complex, but did not behave in a dominantnegative fashion when coexpressed with wild-type caveolin$3{ }^{18}$ Sotgia et al ${ }^{18}$ suggest that differences in caveolin-3 levels and functionality of the remaining wild-type protein may explain varying clinical phenotypes. Since in our patient as well as in reported homozygous and heterozygous RMD patients $^{57}$ caveolin-3 was almost completely absent, additional modifying factors may explain the phenotypic differences. It is becoming clear that RMD is the predominant caveolinopathy phenotype because presently around 100 RMD and around 20 LGMDIC patients have been reported. However, the present data suggest that mutations involving the scaffolding domain lead to the most severe phenotype (LGMDIC). In LGMDIC, inhibited signalling has been documented with regard to neuronal nitric acid synthase (nNOS) and caveolar lipid raft domains (for a review see Razani et $\left.a l^{6}\right)$. In contrast to decreased nNOS expression in LGMDIC, increased inducibility of nNOS has been documented in heterozygous and homozygous RMD. ${ }^{45}$ Furthermore, caveolin-3 is strongly localized to the neuromuscular junction in rat skeletal muscle fibres. ${ }^{19}$ Therefore, severe loss of caveolin-3 and increased nNOS expression could modify the signal transduction cascade, which may explain, at least in part, mechanical hyperirritability of muscle in RMD.

\section{ACKNOWLEDGEMENTS}

We thank I Goebel and V Van den Berghe for expert technical assistance.

\section{Authors' affiliations}

P Y K Van den Bergh, Centre de Référence Neuromusculaire, Cliniques universitaires St-Luc, Université catholique de Louvain, Brussels, Belgium 
J M Gérard, J A Elosegi, Service de Neurologie, Hôpital Ambroise Paré, Mons, Belgium

M U Manto, Servive de Neurologie, Hôpital Erasme, Université Libre de Bruxelles, Brussels, Belgium

C Kubisch, Institute of Human Genetics, University of Bonn, Bonn, Germany

B G H Schoser, Friedrich-Baur-Institute, Deparment of Neurology, Ludwig-Maximilians-University, Munich, Germany

CK was funded by the Deutsche Forschungsgemeinschaft (DFG)

Competing interests: none declared

Presented at the $8^{\text {th }}$ World Muscle Society Congress in Szeged, Hungary, September 3-6, 2003

Correspondence to: P Y K Van den Bergh, MD, PhD, Centre de Référence Neuromusculaire, Cliniques universitaires St-Luc, Université catholique de Louvain, 10 avenue Hippocrate, 1200 Brussels, Belgium; vandenbergh@nchm.ucl.ac.be

Received 13 September 2003

In revised form 3 December 2003

Accepted 5 December 2003

\section{REFERENCES}

1 Torbergsen T. A family with dominant hereditary myotonia, muscular hypertrophy, and increased muscular irritability, distinct drom myotonia congenital Thomsen. Acta Neurol Scand 1975;51:225-32.

2 Ricker K, Moxley RT, Rohkamm R. Rippling muscle disease. Arch Neurol 1989:46:405-8.

3 Vorgerd $\mathrm{M}$, Bolz H, Patzold T, et al. Phenotypic variability in rippling muscle disease. Neurology 1999;52:1453-9.

4 Betz RC, Schoser BGH, Kasper D, et al. Mutations in CAV3 cause mechanical hyperirritability of skeletal muscle in rippling muscle disease. Nat Genet 2001;28:218-19.
5 Kubisch C, Schoser BGH, von Düring $M$, et al. Homozygous mutations in Caveolin-3 cause a severe form of Rippling Muscle Disease. Ann Neurol 2003;53:512-20.

6 Razani B, Woodman E, Lisanti MP. Caveolae: from cell biology to animal physiology. Pharmacol Rev 2002;54:431-67.

7 Vorgerd M, Ricker K, Ziemssen F, et al. A sporadic case of rippling muscle disease caused by a de novo caveolin-3 mutation. Neurology 2001;57:2273-7.

8 Fischer D, Schroers A, Blümke I, et al. Consequences of a novel caveolin-3 mutation in a large German family. Ann Neurol 2003;53:233-41.

9 Merlini L, Carbone I, Capanni C, et al. Familial isolated hyperCKaemia associated with a new mutation in the caveolin-3 (CAV-3) gene. J Neurol Neurosurg Psychiatry 2002;73:65-7.

10 Minetti C, Sotgia F, Bruno C, et al. Mutations in the caveolin-3 gene cause autosomal dominant limb-girdle muscular dystrophy. Nat Genet 1998;18:365-8.

11 McNally EM, de Sá Moreira E, Duggan DJ, et al. Caveolin-3 in muscular dystrophy. Hum Mol Genet 1998;7:871-7.

12 Herrmann R, Straub V, Blank M, et al. Dissociation of the dystroglycan complex in caveolin-3-deficient limb girdle muscular dystrophy. Hum Mol Genet 2000;9:2335-40.

13 Matsuda C, Hayashi YK, Ogawa M, et al. The sarcolemmal proteins dysferlin and caveolin-3 interact in skeletal muscle. Hum Mol Genet 2001; 10:1761-6.

14 Figarella-Branger D, Pouget J, Bernard JR, et al. Limb-girdle muscular dystrophy in a 71-year-old woman with an R27Q mutation in the CAV3 gene. Neurology 2003;61:562-4.

15 Carbone I, Bruno C, Sotgia F, et al. Mutation in the CAV3 gene causes partial caveolin-3 deficiency and hyperCKemia. Neurology 2000;54:1373-6.

16 Tateyama M, Aoki M, Patzold T, et al. Mutation in the caveolin-3 gene causes a peculiar form of distal myopathy. Neurology 2002;58:323-5.

17 Yabe I, Kawashima A, Kikuchi S, et al. Caveolin-3 gene mutation in Japanese with rippling muscle disease. Acta Neurol Scand 2003;108:47-51.

18 Sotgia F, Woodman SE, Bonuccelli G, et al. Phenotypic behavior of caveolin-3 (R26Q), a mutant associated with hyperCKemia, distal myopathy, and rippling muscle disease. Am J Physiol Cell Physiol 2003;285:C1150-60.

19 Carlson BM, Carlson JA, Dedkov El, et al. Concentration of caveolin-3 at the neuromuscular junction in young and old rat skeletal muscle fibers. J Histochem Cytochem 2003;51:1113-18. 